\title{
CONSIDÉRATIONS SUR LE MÉCANISME CHIMIOSENSORIEL DE LA MIGRATION REPRODUCTRICE CHEZ LES SALMONIDÉS
}

\author{
Ph. SAGLIO*
}

* Laboratoire d'Ecologie des Poissons - Station d'Hydrobiologie - I.N.R.A., BP 3, Saint-Pée-surNivelle, 64310 ASCAIN, France.

\section{RÉSUME}

En réponse au problème posé par le rôle du sens chimique dans la migration reproductrice des salmonidés, deux hypothèses ont été particulièrement développées ces dernières décennies :

- L'hypothèse d'HASLER ("Hypothèse de l'imprégnation"), selon laquelle le retour des reproducteurs jusqu'aux sites originels de développement dépend d'un processus de mémorisation à support olfactif mis en place au stade jeune.

- L'hypothèse de NORDENG ("Hypothèse phéromonale") qui considère que l'orientation des reproducteurs est assurée par la perception olfactive de substances attractantes en provenance des "smolts" et des "parrs" de même population.

En dépit de considérations divergentes sur l'influence du facteur génétique dans le phénomène, les démonstrations dont ces axes théoriques ont fait l'objet permettent de considérer que les deux processus sont impliqués dans le "homing" des salmonidés. Les résultats des travaux menés à l'appui de ces hypothèses sont présentés et discutés.

Par ailleurs, nous soulignons le caractère probable de l'action de substances organiques extra-phéromonales dans le "homing". Enfin, nous considérons que les acides aminés libérés par les organismes du milieu pourraient jouer un rôle à la fois dans les orientations phéromonales et allélochimiques des juvéniles et dans le processus d'imprégnation olfactive de ces poissons.

\section{ABSTRACT \\ CONSIDERATIONS ON THE ROLE OF CHEMICAL SENSE IN HOMING OF SALMONIDS.}

Within last decades, two hypotheses have evolved in reply to the problem set by the role of chemical sense in homing of Salmonids :

- HASLER's hypothesis ("Imprinting hypothesis") states that early memorization of stream odor is a prerequisite to homeward migration.

- NORDENG's hypothesis ("Pheromone hypothesis") considers that homeward migration is contralled by olfactive perception of attractive chemicals derived from the related smolt and parr. Despite of divergent opinions about genetic influence, demonstrations issued from those two basic theories support the assumption that both processes are involved in homing of salmonids.

After presentation and discussion of evidence supporting those hypotheses, we stress the probable action of non pheromonal compounds in homing.

Lastly, we consider that free amino acids released by other organisms in the initial environment could not only play a role in pheromonal and allelochemical orientations at the juvenile stage but also contribute to the imprinting mecanism.

\section{1 - POSITION DU PROBLEME}

La migration se définit succinctement par des déplacements orientés entre des sites d'alimentation (ou d'engraissement) et des sites de reproduction.

Au sein des canaux sensoriels impliqués dans les mouvements migratoires des Salmonidés des genres Salmo et Oncorhynchus (consulter STASKO. 1971 : STASKO et al., 1973), l'olfaction joue 
un rôle prépondérant dans l'orientation au cours de la phase finale de la migration de reproduction, depuis les eaux côtières jusqu'aux sites de fraie de l'affluent originel ("homing").

En dépit de l'intérêt des hypothèses avancées ces trois dernières décennies sur le mécanisme chimiosensoriel du "homing" des salmonidés, l'exclusivisme de certaines théories, la variété des espèces utilisées, le faible niveau de dévelopement des mesures éthologiques et les problèmes posés par l'expérimentation dans un milieu peu maîtrisable (ou modélisable) ont contribué à retarder considérablement l'identification chimique des substances naturelles actives sur l'orientation des adultes, des èaux côtières aux zones de frayères.

II demeure aujourd'hui une absence de données :

- sur l'origine et sur la nature précises des substances naturelles impliquées dans le conditionnement olfactif précoce soutenu par HASLER (1966) ;

- sur l'identité chimique et sur l'action comportementale dans le milieu naturel des substances d'origine intraspécifique ("phéromones"), responsables selon NORDENG (1971) de l'orientation des reproducteurs jusqu'aux zones de frayère originelles :

- sur 1 action eventuelle de composés d'origines alimentaire et interspécifique dans le phénomène migratoire.

Au travers d'un nouveau bilan des connaissances sur la communication chimique chez les Salmonidés, nous examinerons ici les substances naturelles (organiques, minérales) pouvant agir sur l'olfaction et sur l'orientation de ces poissons. Le cas du saumon atlantique sera plus particulièrement développé dans cet exposé.

\section{2 - DONNEES PRÉlIMINAIRES SUR LE “HOMING"}

\section{- Attachement au site}

Comme tous les Salmonidés migrateurs, le saumon atlantique se caractérise par un attachement au site originel de développement. Bien que ce phénomène constitue un trait comportemental commun à de nombreux animaux, il apparaît comme un fait particulièrement remarquable chez cette espèce, capable de retourner frayer dans le cours d'eau originel après un voyage océanique pouvant atteindre plusieurs milliers de kilomètres.

Dès 1903, des expériences de marquage de "smolts" sauvages (issus de la rivière TAY, Ecosse) mirent en évidence la fidélité de cette espèce vis-à-vis du cours d'eau natal (CALDERWOOD, 1922).

Plus récemment, SAUNDERS (1967) montra que le "homing" du saumon atlantique à l'intérieur de la rivière Miramichi (Canada) s'achevait précisément dans les mêmes zones que celles qu'ils occupaient à l'état de juvenile ("parr"). Ceci révèle l'extrême précision des moyens d'orientation utilisés par le poisson, au cours de la phase finale de la migration reproductrice.

\section{- Possibilité d'induction du phénomène}

WHITE (1936) démontra que l'introduction du saumon atlantique à l'état d'alevins dans un affluent non colonisé d'une petite rivière canadienne donnait lieu à des remontées d'adultes dans les années suivantes.

Cette possibilité d'induction du "homing" a été confirmée depuis par les résultats des nombreuses expériences de transplantations réalisées au Canada (STASKO et al., 1973), en Norvège (ROSSELAND, 1978) et en Islande (ISAKSSON et al., 1978).

Toutefois, le taux de retour de ces "transplants", issus d'œufs artificiellement fécondés ou de "śmolts" d'élevage, apparaît le plus souvent inférieur à celui présenté par les poissons natifs du système fluvial étudié.

En Norvège, les expériences de transplantations réalisées de 1957 à 1962 ont donné un taux moyen de retour de $0,09 \%$ contre $2,3 \%$ pour les populations sauvages étudiées au cours de la même période. Par ailleurs, le taux de divagation (1) des poissons nouvellement introduits demeure très supérieur à celui présenté par les poissons sauvages.

Le retour du saumon atlantique après transplantation devrait pouvoir être amélioré par la mise en pratique des nombreuses connaissances accumulées ces 20 dernières années d'une part sur les facteurs génétique, physiologique et environnemental susceptibles d'action sur le développement de ce poisson (présentées par SAUNDERS, 1977, 1982 ; SAUNDERS et BAILEY, 1980 ; PIGGINS et BROWNE, 1982) et d'autre part, sur les mécanismes d'orientation impliqués dans le "homing".

(1) Exprimé par le pourcentage de recaptures dans d'autres rivières que la rivière expérimentale. 


\section{3 - PREMIERES HYPOTHESES}

Bien que l'hypothèse du rôle de l'olfaction dans le "homing" date de plus d'un siècle (BUCKLAND, 1880), elle ne recut que tardivement un soutien expérimental et d'autres hypothèses accordant une place essentielle aux caractéristiques physico-chimiques de l'eau ont été for mulées. Ainsi, celles de WARD (1939) et de HOAR (1958) selon laquelle la perception par le saumon de gradients thermiques permettent son orientation jusqu'a la rivière originelle. Selon POWERS et CLARK (1943) et COLLINS (1952), le paramètre responsable serait la tension en gaz carbonique de l'eau.

Sans écarter leur possibilité d'action au cours de la migration reproductrice, du fait de leur effet sur le rhéotropisme (NORTHCOTE, 1962) et sur l'orientation des Salmonidés, ces paramètres restent insuffisants selon SCHEER (1939) et HASLER (1971), pour rendre compte de la remarquable spécificité du choix effectué par le saumon vis-à-vis de son affluent originel.

\section{4 - MISE EN ÉVIDENCE DU ROLE DE L'OLFACTION}

Chez le saumon atlantique, la preuve de la validité de l'hypothèse olfactive émise par BUCKLAND à la fin du siècle dernier ne fut apportée que récemment par certaines expériences de privation sensorielle pratiquées dans le milieu naturel.

Après lésions bilatérales des nerfs olfactifs de "grilses" de saumons de la Baltique (Salmo salar) capturés au moment de leur remontée fluviale (rivière Indalselven, Suède) et déplacés à près de $40 \mathrm{~km}$ de l'estuaire. BERTMAR et TOFT (1969) constatèrent que ces poissons étaient pour la plupart incapables de retourner jusqu'à la rivière. Dans des études complémentaires, TOFT (1975) confirma ce fait et montra par ailleurs qu'une privation totale de la vision ou unilatérale de l'olfaction affectait peu le retour des reproducteurs, après déplacement à $100 \mathrm{~km}$ de l'estuaire.

Outre cette espèce, de nombreuses expériences de lâchers-recaptures de poissons porteurs de lésions olfactives ou/et visuelles montrent que l'olfaction constitue un canal sensoriel dont l'activité est déterminante dans cette structure de comportement que représente le "homing" des salmonidés (voir revue établie par STASKO, 1971).

\section{5 - THEORIES EN COURS}

Au cours de la de uxième partie de ce siècle, les recherches sur les bases chimiosensorielles de la migration reproductrice des salmonidés se sont développées principalement selon deux axes thériques : la première hypothèse introduite par HASLER et WISBY (1951 (1), considère que le "homing" repose sur les capacités de conditionnement olfactif précoce des poissons, tandis que celle de NORDENG (1971 (2)) accorde aux interactions chimiques à caractère intra-spécifique ("interactions phéromonales") un rôle essentiel dans cette phase d'orientation.

Nous examinerons brièvement l'intérêt de ces deux hypothèses, à la lueur des appuis expérimentaux dont elles disposent aujourd'hui.

\subsection{Conditionnement olfactif précoce}

\subsubsection{Théorie d'HASLER : définition et bases expérimentales}

Selon HASLER (1966), chaque rivière est constituée d'un bouquet d'odeurs caractéristiques et l'orientation sélective des reproducteurs vis-à-vis de celle-ci est assurée grâce à un phénomène de conditionnement olfactif opérant au stade jeune.

Cette théorie de l'imprégnation ("imprinting hypothesis") implique à la fois un haut pouvoir de discrimination olfactive et l'existence d'une mémoire à long terme. Se basant sur les résultats de nombreux travaux (résumés par HASLER et COOPER, 1976 ; HASLER et al., 1978), menés par le Laboratoire de Limonologie de l'Université de Wisconsin (USA), HASLER présente les preuves suivantes à l'appui de son hypothèse :

- la haute sensibilité olfactive des poissons susceptibles de détecter des substances à des concentrations de l'ordre de $10^{-6} \mathrm{mg} / \mathrm{l}$ :

- la capacité de conditionnement olfactif à des eaux de différentes origines :

(1) Hypothèse surtout développée chez des espèces du genre Oncorhynchus.

(2) Hypothèse développée chez des espèces du genre Salmo. 
- les retours de reproducteurs constatés après transplantation d'œufs ou de juvéniles sur de nouveaux sites :

- le rôle actif de l'olfaction dans l'orientation, mis en évidence par les expériences de privations sensorielles partielles ;

- les réactions comportementales (olfactométrie) et les modifications de la sensibilité des récepteurs olfactifs (électrophysiologie sensorielle) observées en réponse à un effluent d'eau provenant du site originel de développement :

- la possibilité d'utiliser un composé artificiel afin de faire migrer sélectivement les adultes dans un site donné, après imprégnation à ce composé au moment de la smoltification :

- les suivis radiotélémétriques de déplacements des reproducteurs à proximité du site d'imprégnation artificielle.

\subsubsection{Intérêts et limites de l'approche utilisée}

L'hypothèse d'HASLER est étayée d'observations remarquables qui révèlent d'une part le rôle prépondérant de l'olfaction dans le "homing" et d'autre part l'existence d'une mémoire à long terme édifiée à partir des caractéristiques physico-chimiques de l'environnement percu au stade jeune.

Outre son intérêt appliqué immédiat, l'orientation méthodologique soutenant cette hypothèse donne accès à l'étude fine de nombreux aspects du mécanisme chimiosensoriel de la migration chez les Salmonidés.

Toutefois, la plupart des travaux ont été menés sur le saumon "coho" (Oncorhynchus kisutch) dans lès grands lacs américains. Chez le saumon atlantique, les bases de cette théorie d'une imprégnation olfactive précoce suivie de mémorisation à long terme demeurent inexplorées, ce qui rend pour l'instant hasardeuse toute tentative d'imprégnation artificielle appliquée au sea-ranching de cette espèce. remarques :

Par ailleurs, l'approche utilisée par l'équipe d'HASLER suscite un certain nombre de

- Peu de données sur l'environnement et sur les substances naturelles impliquées dans "I'imprégnation" au stade jeune.

Dans l'hypothèse d'HÀSLER, la nature des substances actives sur l'imprégnation précoce dépendrait des composantes odorantes du milieu originel en relation avec le sol, la flore et la faune en place.

Bien que le "bouquet odorant" résultant de ces influences puisse contenir des médiateurs chimiques dont le caractère unique (nature et concentration) s'accorde bien avec la précision du retour des reproducteurs jusqu'au niveau même du site de développement, cette hypothèse n'a été suivie d'aucune étude approfondie sur l'origine, l'effet comportemental ou l'identité chimique des substances naturelles impliquées.

Depuis 1952, date à laquelle WISBY proposait la morpholine $\left(\mathrm{C}_{4} \mathrm{H}_{9}\right.$ NO : P.M. : 87) comme composé artificiel utilisable à des fins de conditionnement olfactif des poissons, l'effort de recherches produit par l'équipe américaine s'est peu dissocié de l'utilisation de ce composé, tant pour la mise en évidence de l'action d'une mémoire à long terme dans le "homing" que pour la définition d'opérations contrôlées dans le cadre du sea-ranching du saumon.

\section{- Faible considération de l'influence génétique.}

La théorie "environnementaliste" d'HASLER fait peu de cas de l'influence du facteur génétique dans la précision du "homing". Cette influence directe a néanmoins été mise en évidence chez le saumon "pink", Oncorhynchus gorbuscha (BAMS, 1976). Chez le saumon atlantique, STABELL (1982 a) souligne également l'importance de ce facteur en faisant remarquer que, malgré des taux de survie comparables au cours de la période de vie marine (1), les populations sauvages présentent un taux de retour jusqu à la rivière originelle supérieur à celui que l'on peut constater chez.des poissons issus de souche d'élevage. En outre, le taux de divagation chez les saumons sauvages est inférieur à celui que l'on peut observer chez des saumons nouvellement introduits. Toutefois, la moindre capacité de "homing" présentée par ces derniers peut également être due à des conditions d'élevage défavorables précédant leur introduction.

(1) Ce fait peut être déduit de l'analyse des données canadiennes de recapture de 1960 à 1970 (dér. de STASKO et al. . 1973). 
A ce sujet, il est regrettable que les études de transplantation et d'imprégnation artificielle pratiquées dans les grands lacs (PECK, 1970 ; MADISON et al., 1973 ; SCHOLZet al., 1975 ; COOPER et al., 1976 ; HASLER et al., 1978) n'aient guère pris en compte l'influence possible des conditions d'élevage avant smoltification (moment du lâcher) sur le taux de retour de ces poissons.

\section{- A propos de la "période critique"' d'imprégnation}

Les travaux de l'école d'HASLER ont permis de démontrer chez le saumon "coho" l'importance du conditionnement olfactif précoce dans le retour des reproducteurs jusqu'au point de lâcher initial au stade smolt.

Selon HASLER et al. (1978) le processus (irréversible) de l'imprégnàtión olfactive est extrêmement rapide et limité à ce stade. Ces auteurs citent l'étude de MIGHELL'(1975) qui mit en évidence que l'exposition de "smolts" de coho pendant 4 heures à l'eau d'une nouvelle rivière suffisait à les faire retourner dans celle-ci à l'état adulte.

De même. CARLIN (1968), après transplantation de saumon atlantique en début de smoltification, constatait que les poissons quittaient la rivière dans les deux jours et revenaient néanmoins $y$ frayer les années suivantes. Bien que ces expériences démontrent une sensibilité particulière des poissons au moment de la smoltification, elles ne permettent pas d'écarter l'action sur le "homing" d'un conditionnement olfactif antérieur à cette période.

Avant le lâcher au stade "smolt", les juvéniles ont été soumis à des conditions,contrôlées parmi lesquelles la qualité de l'eau, la densité en élevage et l'aliment distribué constituent des facteurs susceptibles de créer une sensibilisation olfactive particulière et donc, selon la même hypothèse, d'agir sur l'orientation des comportements des adultes.

Nous pensons qu'il est difficile de soutenir que l'imprégnation olfactive déterminant le "homing" des salmonidés est limitée au stade "smolt" en l'absence de données sur l'action conditionnante éventuelle de ces facteurs chez les juvéniles. En particulier, l'action conditionnante précoce de substances d'origine intraspécifique (phéromones) n'a guère été examinée. Ces phéromones constituent pourtant dans les expériences de transplantation les principaux médiateurs communs à la fois au milieu d'élevage et au milieu de lâcher.

Par ailleurs, ce rapprochement entre smoltification et période d'imprégnation ne peut s'accorder avec la variété des modes migratoires présentés chez les salmonidés. Chez le coho, QUINN (1985) souligne le fait que de nombreux poissons descendent en mer au prémier priritemps à l'état de parr, sans signe apparent de smoltification.

En outre, les études olfactométriques menées par BRANNON (1972) sur des alevins de saumon sockeye (Oncorhynchus nerka) ont révélé l'action possible de conditionnement chimiosensoriel avant l'émergence. De même que chez les pink (O. gorbuscha) et chum (O. keta), le sockeye quitte la zone d'incubation peu après l'émergence.

Chez ces espèces, l'imprégnation devra donc se produire au plus tard au cours de cette période.

\section{- "Imprégnation artificielle" et "sea-ranching"" : des conclusions discutables.}

Dans les expériences types d'imprégnation de saumon "coho" à la morpholine (SCHOLZet al., 1973). l'eau qui alimente les bassins d'élevage des juvéniles (eau de ville) puis des "smolts" (eau pompée à $1,5 \mathrm{~km}$ au large du lac Michigan) est sans relation avec le milieu susceptible d'être occupé par ces poissons aux stades considérés. Dans le lot expérimental (poissons imprégnés). la morpholine constitue, au sein de cet environnement chimique appauvri ou non représentatif, le principal médiateur commun aux deux phases étudiées ("smolt" et géniteur). Par opposition, les poissons du lot témoin, relâchés après smoltification (après la "période critique" d'imprégnation) n'ont pu acquérir aucun type de conditionnement physico-chimique particulier susceptible d'opérer lors de l'orientation ultérieure jusqu' au site de recapture. Ceci est d'autant plus vrai que dans ces expériences d'imprégnation. le site de lâcher (embouchure de la rivière) et le site de recapture (rivière) ne sont pas les mêmes.

Bien que les taux de recapture observés après imprégnation des "smolts" à la morpholine, puis émission de ce composé au moment de la migration reproductrice des adultes plaident en faveur de l'existence d'une mémoire à long terme à support olfactif (1) active sur l'orientation des reproducteurs. l'absence de références simultanées à un lot témoin imprégné aux caractéristiques chimiques du site de lâcher ou de recapture ne permet pas de tirer de conclusions définitives sur l'avantage de cette méthode, par rapport à la seule imprégnation naturelle.

(1) Ce fait peut être également déduit d'une part des résultats des expériences de transplantations et de lésions olfactives et, d'autre part, de l'absence de retour constatée chez les poissons témoins non imprégnés. 
A ce propos, STABELL (1984) montre que les taux de retour observé chez les "smolts" imprégnés à la morpholine sont tout à fait similaires à ceux obtenus à la suite des opérations de transplantations de "smolts" effectuées dans les grands lacs. Il semble donc que chez ces populations d'élevage, l'imprégnation à la morpholine n'augmente en rien le "homing" des reproducteurs, comparée à une imprégnation naturelle au stade "smolt".

Par ailleurs, le choix de la morpholine comme composé d'imprégnation a soulevé de nombreuses critiques (HARA, 1974 ; HARA et MACDONALD, 1975 ; HARA et BROWN, 1979, 1982). Selon ces auteurs, la stimulation des récepteurs olfactifs avec une solution de morpholine produit une réponse électrophysiologique différente (persistance après stimulation) de la réponse normale à des substances odorantes (1) d'activité comportementale. Cette différence serait liée davantage à un effet général irŗitant au niveau de la muqueuse olfactive qu'à une action spécifique au niveau des cellules sensorielles. En outre, la morpholine inhibe la sensibilité olfactive à la L. sérine. Bien qu'en réponse à ces travaux, les études de COOPER et d'HASLER (1976) et de COOPER (1982) apportent la preuve de la relation existant entre la stimulation à la morpholine chez des poissons imprégnés préalablement (à la dose de $5.10^{-5} \mathrm{mg} / \mathrm{l}$ ) et l'apparition de réponses très nettes au niveau des bulbes olfactifs, un certain nombre de problèmes concernant l'activité psychophysiologique de ce composé demeurent posés.

Selon nous, la principale critique que l'on pourrait adresser à la multiplication des expériences d'imprégnation artificielle à la morpholine (2) réside dans le fait que cette méthode risque de perdre très rapidement toute spécificité d'action. Ceci pourrait être évité en disposant d'un plus grand spectre de composés artificiels utilisables (soluble, non toxique, hautement diffusible et perceptible par l'olfaction à faible concentration). Cette approche risque néanmoins d'être très lourde compte tenu de l'importance des études préalables devant être développées en vue de l'utilisation de tels composés.

\subsection{Communications chimiques intraspécifiques}

Les récents développements de la recherche sur la communication chimique intraspécifique chez les salmonidés permettent aujourd'hui de supposer que, parmi les facteurs organiques caractéristiques de l'affluent originel évoqués par HASLER (1 966), les substances émises par les populations en place jouent un rôle essentiel dans l'orientation finale des reproducteurs.

\subsubsection{Définition et historique de l'hypothèse phéromonale}

Dès 1924, CHIDESTER suggérait que le "homing" du saumon atlantique dépendait de la perception chimique par les adultes d" "émanations" provenant des juvéniles présents dans la rivière.

Après implantation d'alevins de saumon atlantique dans l'affluent d'une rivière connue comme ne possédant plus de représentants de cette espèce, WHITE (1936) observait dès la même année d'importantes montaisons d'adultes. Cet auteur émit l'hypothèse que la laitance produite par les mâles précoces de "parr" constituait l'attractant agissant sur la remontée des reproducteurs.

Toutefois, l'absence de précision sur l'origine des alevins introduits laissa cette hypothèse inexplorée jusqu'en 1971, date à laquelle NORDENG démontra que des attractants à caractère racial intraspécifique pouvaient agir sur l'orientation des reproducteurs des populations migratrices d'omble arctique (Salmo alpinus L.).

Dans cette expérience de NORDENG, les œufs artificiellement fertilisés issus de populations migratrices d'omble arctique de la rivière Salangen (Nord de la Norvège) étaient transportés dans une pisciculture du sud de la Norvège où avaient lieu l'éclosion puis l'élevage des poissons pendant 3 ans.

Au terme de ce délai, les poissons étaient relâchés d'une part à l'embouchure de la rivière "parentale" (rivière Salangen) et, d'autre part, à l'embouchure d'une rivière située à $10 \mathrm{~km}$ de celle-ci (rivière Lфksebotn). 26 sur 27 poissons recapturés du $1^{\text {er }}$ groupe (3) furent dénombrés dans la rivière Salangen. Sur les 31 poissons recapturés du $2^{\ominus}$ groupe (4), 21 furent capturés dans la riviere Salangen et 10 dans la rivière L $\varnothing k$ sebotn. Ces résultats montrent que des salmonidés migrateurs ".répondent". préférentiellement à la rivière d'origine parentale, même en l'absence d'expériences précoces des caractéristiques physico-chimiques de l'eau dewelle-ci.

(1) Extraits alimentaires, rinçage de mains, substances muqueuses et acides aminés.

(2) Outre le risque de perturbation des moyens naturels d'orientation chimique.

(3) Lâcher initial de 143 poissons à l'estuaire Salangen.

(4) Lâcher initial de 174 poissons à l'estuaire de Løksebotn. 
Chez le saumon atlantique, SOLOMON (1973) souligne la rareté des captures de poissons à proximité d'estuaires de rivières non colonisées par l'espèce, par opposition au mélange des stocks constatés à proximité de rivières colonisées. Selon l'auteur, la présence de juvénilés de saumon atlantique dans une rivière produit une attraction générale des adultes à proximité de son embouchure.

SOLOMON cite pour preuve que l'introduction d'cufs de saumon atlantique dans un affluent de la rivière Parret (Grande-Bretagne) a permis d'augmenter considérablement les captures à proximité de l'estuaire de cette rivière.

L'intervention de phéromones de caractéristiques plus spécifiques (effet au niveau de la. population) sur la dernière phase du "homing". ne peut cependant être déduite des observations de SOLOMON.

Près de 20 années d'observations sur les déplacements migratoires des populations de saumon atlantique, d'omble arctique et de truite, Salmo trutta L., à l'intérieur de la rivière Salangen, permirent à NORDENG (1977) d'édifier une hypothèse susceptible dans son ensemble de rendre compte de la précision du "homing" chez les salmonidés migrateurs.

L'hypothèse phéromonale de NORDENG s'appuie sur deux faits essentiels, constatés chez les trois espèces étudiées:

a) les "smolts" ont commencé leur migration aval quelque temps avant que les premiers adultes de la même espèce ne soient arrivés à l'embouchure.

b) les mouvements de migration aval des smolts et de migration amont des adultes se superposent pendant une période spécifique et un "trafic" à deux voies s'instaure alors dans la rivière et dans les eaux proches de l'estuaire.

Considérant ces faits, l'orientation des adultes jusqu'à l'embouchure de la rivière s'effectuerait grâce à la perception chimique de substances phéromonales émises simultanément par les "smolts" de mêmes populations au cours de leur dévalaison et de leur migration (dans le sens du courant, BRAEMER, 1960 ; ROYCE et al., 1968) vers les sites d'engraissement. L'orientation finale jusqu'aux sites de fraie pourrait être déterminée par la présence de phéromones venant à la fois des populations de jeunes en place ("parr") et des premiers reproducteurs arrivés. Dans le cas particulier du saumon pink (Oncorhynchus gorbuscha), dont la remontée migratoire s'effectue dans des rivières alors dépourvues d'individus de la même population (cycle bisannuel), NORDENG suggère la possibilité d'une communication chimique avec les autres saumons du Pacifique (O. keta et O.nerka) présents dans ces rivières, tandis que selon LARKIN (1975), l'orientation des reproducteurs de cette espece serait assurée grâce à la perception de substances odorantes laissées par le "run" précédent. Chez d'autres saumons du genre Oncorhynchus (O. nerka, kisutch et tshawytscha), LARKIN émit également l'hypothèse selon laquelle les odeurs intraspécifiques provenant des populations en place constitueraient les facteurs chimiques responsables de l'orientation des reproducteurs.

\subsubsection{Différents types d'interactions phéromonales}

Dans I'hypothèse de NORDENG, l'action attractive très spécifique (1) de substances provenant d'une part des "smolts" en mouvement et d'autre part des populations en place de juvéniles ("parr") permettrait aux adultes de s'orienter jusqu'aux sites originels.

Outre ces interactions, des communications phéromonales entre juvéniles ou entre reproducteurs peuvent également être impliquées dans le "homing".

Avant de définir les substances actives, nous examinerons ici les principaux modes de communication chimique intraspécifique mis en évidence chez les Salmonidés.

\section{- Interactions aux stades "parr" et "smolt"}

Chez le saumon atlantique, les retours de "parr" après déplacement à l'intérieur de la rivière. originelle(SAUNDERS et GEE, 1964) prouvent que l" aptitude au "homing" se développe précocement. Par ailleurs, le fait que la migration reproductrice des adultes s'achève précisément au même site que celui occupé à l'état de "parr" (SAUNDERS, 1967) montre que les interactions sensorielles à ce stade sont susceptibles d'agir sur l'orientation des comportements ultérieurs.

En 1973, HOGLUND et ASTRAND démontrèrent en "fluvarium" (2) l'existence d'interattractions phéromonales chez les juvéniles d'omble arctique et mirent en évidence le rôle de l'olfaction dans le phénomène.

Des interactions phéromonales semblent également opérer entre "parr" de saumon atlantique (STABELL, 1982 b).

(1) Action limitée aux poissons de mêmes populations.

(2) Olfactomètre type ruisseau artificiel à choix multiple. 
Chez le saumon coho, les interattractions se produisent préférentiellement entre juvéniles de même parenté et un conditionnement collectif préalable renforce le caractère attractif des substances impliquées (QUINN et BUSACK, 1985).

La possibilité d'une odeur spécifique à chaque population est ici suggérée par le fait que, même en l'absence d'expérience commune préalable, l'attraction est plus importante en réponse aux juvéniles de même parenté qu'en réponse à des juvéniles d'autres souches.

L'effet de la perception phéromonale de substances émises par les reproducteurs sur le comportement des "parr" ou des "smolts" demeure pour l'instant inconnu. Il en est de même des interrelations phéromonales éventuelles entre "smolts".

\section{- Interactions au stade reproducteur}

Les substances phéromonales actives sur les reproducteurs peuvent provenir des juvéniles, des "smolts" ou des reproducteurs eux-mêmes.

\section{a) Perception phéromonale des juvéniles par les reproducteurs.}

Deux observations soulignent l'attraction intraspécifique exercée par-les jeunes avant smoltification sur les reproducteurs.

- Au moment de la migration reproductrice de saumons d'élevage et de saumons sauvages à I'intérieur de la rivière St-John, (New Brunswick, Canada), SUTTERLIN et GRAY (1973) constatèrent que $67 \%$ des poissons d'élevage étaient retournés à l'écloserie contre $3 \%$ seulement pour les poissons sauvages. Des tests olfactométriques (1) complémentaires confirmèrent la préférence des adultes d'élevage pour l'eau issue de l'écloserie, tandis que celle-ci demeurait sans action comportementale sur les poissons sauvages.

- Ce fait peut être rapproché de l'étude de FOSTER et BERLIN (1980) qui démontra que le marquage du substrat avec des extraits réalisés à partir d'alevins de truite de lac. Salvelinus namaycush. orientait le choix.du site de fraie chez les reproducteurs de cette espèce.

b) Perception phéromonale des smolts par les reproducteurs.

Chez l'omble arctique à maturité, les enregistrements électrophysiologiques des réponses olfactives provoquées par la stimulation avec de l'eau ayant contenu des "smolts" mirent en évidence la sensibilité phéromonale de cette espèce (DØVING et al., 1973).

Dans une étude complémentaire. DøVING et al. (1974) montrèrent la capacité de discrimination olfactive de l'adulte vis-à-vis de "smolts" de différentes populations. Cette capacité de discrimination fut confirmée par les études comportementales en olfactomètre réalisées par SELSET et DOVING (1980).

Chez le saumon atlantique, les mesures électrophysiologiques effectuées par FISKNES et D $\phi$ VING (1982) soulignent également la sensibilité olfactive particulière des poissons matures visa-vis des "smolts". Cependant, l'effet spécifique lié à la population étudiée ne semble pas aussi net que chez l'omble arctique.

\section{c) Perception phéromonale entre reproducteurs}

L'action de phéromones sexuelles sur les inter-relations entre reproducteurs a été mise en évidence chez de nombreuses espèces de poissons (consulter LILEY, 1982).

Encore peu explorée chez le saumon atlantique, cette action semble néanmoins courante chez les salmonidés. Chez la truite arc-en-ciel, Salmo gairdneri, NEWCOMBE et HARTMAN (1973) révélèrent l'existence d'interattractions sexuelles entre reproducteurs à maturité.

L'attraction exercée sur le mâle mature par la femelle ovulée de cette espèce fut ensuite démontrée par EMANUEL et DODSON (1979), puis par HONDA (1980) qui montra que l'olfaction était déterminante dans la réalisation du comportement sexuel du mâle.

L'attraction en olfactomètre du mâle par la femelle de la même espèce ressort également des observations de PETE (1977) chez le Coho et chez le Chinook (O. tshawytscha) et de DUKER (1981) chez le Chum (O. keta).

Chez les mâles de saumon du genre Oncorhynchus (O. masou et $O$. rhodorus) HONDA (1982) mit en évidence la capacité de discrimination olfactive du cycle sexuel de la femelle. L'induction du comportement sexuel des mâles de chacune de ces espèces apparaît étroitement liée à la perception olfactive des femelles respectives, au stade post-ovulatoire.

(1) Système à double choix. 


\subsubsection{Substances actives}

L'épiderme, le système digestif et les gonades constituent à ce jour les trois principales origines histophysiologiques des substances responsables des interactions phéromonales chez les salmonidés.

\section{- Mucus épidermique}

Chez les poissons, le mucus épidermique constitue un médiateur chimique d'importance dans les phénomènes de socialisation intraspécifique.

L'action phéromonale attractive de cette sécrétion superficielle a été démontrée au sein des Cyprinidés (WREDE, 1932; HATTINGH et COETZEE, 1975; SAGLIO et LE MARTRET, 1982), des Ictaluridés (TODD et al., 1967) et des Anguillidés (SAGLIO, 1982).

Chez les salmonidés migrateurs, NORDENG (1971) suggérait que le mucus épidermique des juvéniles et des "smolts" possédait un effet attractif sur les reproducteurs de même origine.

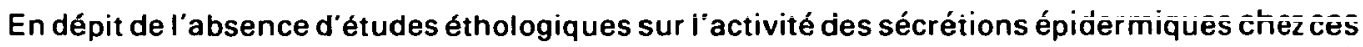
poissons, les résultats des mesures électro-physiologiques réalisées au niveau des voies olfactives à partir d'extraits bruts ou de fractions d'analyses du mucus soutiennent cette possibilité.

L'activité olfactive de cette sécrétion fut tout d'abord mise en évidence par D $\emptyset$ VING et al. (1973) chez l'o mble arctique. L'activité observée en réponse au mucus épidermique apparaissait supérieure à celle provoquée par des extraits intraspécifiques d'urine, de laitance, de sang ou de muscle.

Par ailleurs, l'importance de cette sécrétion dans la perception des caractéristiques odorantes de l'espèce était suggérée par le fait que les extraits de mucus épidermique de juvéniles d'omble arctique présentaient un effet stimulant sur les voies olfactives des adultes, très proche de celui produit par l'eau ayant contenu ces juvéniles.

Chez le saumon atlantique, STABELL et al. (1982) relevèrent des différences dans la composition chimique du mucus épidermique de juvéniles de saumon atlantique provenant de trois rivières de Norvège. FISKNES et D $\phi$ VING (1982) confirmèrent l'activité olfactive du mucus épidermique chez cette espèce. Toutefois, l'activité observée en réponse aux fractions isolées par STABELL n'apparut pas spécifique des populations étudiées.

L'activité du mucus épidermique sur l'olfaction des Salmonidés a été également démontrée chez la truite arc-en-ciel et chez un corégone, Coregonus clupeaformis (HARA et MAC DONALD. 1975 ; HARA et al., 1984). Chez la truite arc-en-ciel, HARA et al. (1973) montrèrent que le poids moléculaire des substances présentes dans le mucus et actives sur l'olfaction était inférieur à 1000. Parmi les composés de faible poids moléculaire présents dans le mucus épidermique et susceptibles d'activité chimiosensorielle chez les salmonidés, des acides aminés libres avaient été isolés par STABELL et SELSET (1980) chez l'omble arctique.

Les récentes analyses électrophysiologiques et biochimiques réalisées par HARA et al. (1984) indiquent que les acides aminés libres du mucus sont responsables de l'activité de la séćrétion au niveau des voies olfactives.

Chez les deux espèces citées ci-dessus. HARA et al. (1984) montrèrent que la fraction d'acides aminés libres du mucus épidermique présentait le même caractère stimulant au niveau des bulbes olfactifs que la sécrétion brute. Les auteurs mirent par ailleurs en évidence des différences dans la teneur totale en acides aminés libres en fonction de l'espèce considérée.

Ces travaux soulignent l'intérêt de recherches ultérieures sur l'activité comportementale des acides aminés libres présents dans les sécrétions épidermiques superficielles des poissons.

\section{- Produits d'excrétion}

De même que chez de nombreux vertébrés (consulter MULLER-SCHWARZE et MOZELL, 1977), les produits d'excrétion constituent chez les poissons une source de médiateurs d'actions chimiosensorielle et comportementale intraspécifique.

Chez les Salmonidés, les travaux norvégiens réalisés ces cinq dernières années ont révélé le caractère hautement stimulant à la fois sur les voies olfactives et sur le comportement de substances d'origine intestinale.

SELSET et D $\varnothing V I N G$ (1980) montrèrent en olfactomètre à double choix que les reproducteurs d'omble arctique étaient attirés par des extraits de mucus intestinal de "smolts".

L'attraction observée en réponse à ces extraits apparaissait supérieure à celle produite par le mucus épidermique de ces poissons ou par des extraits alimentaires.

Par ailleurs, cette attraction présente un caractère très spécifique puisque les reproducteurs étaient préférentiellement attirés par des extraits intestinaux provenant de "smolts" de la même population. 
Après analyse biochimique du mucus intestinal de ces poissons (SELSET et D $\emptyset$ VING, 1980) et tests complémentaires, il apparut que des composés apparentés à des acides biliaires étaient responsables de l'activité comportementale observée.

Chez les salmonidés, les acides biliaires produits sont principalement les acides choliques et chénodésoxycholiques conjugués à la taurine (HASLEWOOD, 1967 ; DENTON et al., 1975 ; SACQUET et al., 1979). D $\varnothing$ VING et al. (1980) montrèrent chez des adultes d'omble, Salmo alpinus et d'ombre arctique, Thymallus thymallus, que le seuil olfactif de perception de ces composés était extrêmement bas $\left(10^{-9} \mathrm{M}\right)$.

Les récents travaux électrophysiologiques de HARA et al. (1984) ont montré que la gustation était aussi impliquée dans la perception des acides biliaires. Ces auteurs recueillirent des réponses gustatives à des doses de $10^{-12}$ a $10^{-11} \mathrm{M}$ d'acide taurolithocholique.

Chez le saumon atlantique, STABELL et al. (1982) mirent en évidence des différences dans la composition chimique du mucus intestinal de juvéniles en fonction de l'origine des poissons étudiés. Toutefois, les tests d'activité électrophysiologique réalisés au niveau des bulbes olfactifs de l'adulte par FISKNES et D $\phi$ VING (1982) à partir des analyses de STABELL ne révélèrent pas chez cette espèce de réponses limitées aux poissons de mêmes populations. De même que pour le mucus épidermique, l'activité du mucus intestinal ne paraît donc pas aussi spécifique chez le saumon atlantique que chez l'omble arctique.

Bien que la présence d'acides biliaires dans les excreta des salmonidés (SACQUET et al., 1979) puisse constituer des marques odorantes utilisables par ces poissons, l'action phéromonale des fèces demeure peu étudiée.

De telles phéromones semblent néanmoins exister, ainsi que l'indique chez la truite de lac l'action de feces d'alevins sur le choix du site de fraie effectué par les reproducteurs (FOSTER et BERLIN, 1980).

Comme pour les fèces, l'action éventuelle de l'urine dans la relation intraspécifique des salmonidés n'a guère été étudiée. Peu stimulante sur les voies olfactives de l'omble arctique (D $\phi$ VING et al., 1973), apparemment très active sur l'olfaction du saumon atlantique (FISKNES et D $\emptyset$ VING, 1982), le rôle de l'urine dans les interactions phéromonales de ces poissons reste à préciser.

\section{- Produits sexuels}

Chez les Salmonidés, des interattractions chimiques entre poissons matures jouent un rôle déterminant dans la synchronisation des comportements reproducteurs mâle et femelle. De telles interactions ont été décrites par NEWCOMBE et HARTMAN (1973) chez la truite arc-en-ciel.

Au sein des attractions intersexuelles observées chez cette espèce, les auteurs montrèrent que les femelles étaient plus attirées par les mâles que ces derniers pouvaient l'être par les femelles.

L'émission par le mâle de substances aphrodisiaques provoquant l'attraction de la femelle n'a guère été confirmée chez les autres salmonidés étudiés.

Ce type d'attraction sexuelle (femelle par mâle) existe néanmoins chez de nombreuses espèces de poissons et les substances impliquées peuvent être présentes dans l'urine (RUBEC, 1979), dans les gonades (OKADA et al., 1978), dans le fluide urogénital (TEETER, 1980) ou dans le sperme (STACEY et HOURSTON, 1982).

Compte tenu des différences relevées entre mâle et femelle dans les teneurs en acides aminés libres du mucus épidermique chez la truite arc-en-ciel et le corégone (Coregonus c/upeaformis) (HARA et al., 1984), cette sécrétion pourrait également être impliquếe dans des processus d'identification sexuelle.

Chez la truite arc-en-ciel, EMANUEL et DODSON (1979) mirent en évidence l'effet attractif sur le mâle mature du liquide ovarien de la femelle. En olfactomètre, cet effet apparaît maximum à la concentration de $10^{-3} \mu \mathrm{l} / \mathrm{I}$. HONDA (1980) confirma l'attractivité sexuelle exercée sur le mâle par la femelle de truite arc-en-ciel et montra que la phéromone était émise dans la cavité coelomique avec les œufs (mais non dans les œufs), aussitôt après l'ovulation. Une médiation phéromonale sexuelle du même type fut révélée par HONDA (1982) chez Oncorhynchus masou et $O$. rhodorus.

Bien que l'attractivité de la femelle ovulée sur le mâle mature ait été décrite chez nombre de poissons (consulter LILEY, 1982), l'identité chimique des médiateurs impliqués demeure inconnue. II en est de même des substances aphrodisiaques émises par les mâles.

Néanmoins, du fait de leur action limitée à la période de maturité sexuelle des poissons, ces phéromones apparaissent étroitement dépendantes de contrôles endocriniens. 
L'action d'hormones stéroïdes sur la sensibilité olfactive a été mise en évidence par HARA (1967), puis par OSHIMA et GORBMAN (1968) chez le carassin doré, Carassius auratus.

Tout comme chez la truite, le mâle du carassin doré est attiré par la femelle ovulée et la phéromone est présente par le fluide ovarien (PARTRIDGE et al., 1976).

Or, YAMAZAKI et WATANABE (1979) montrèrent chez cette dernière espèce que les mâles matures hypophysectomisés et traités avec des œstrogènes présentaient le même caractère attractif que les femelles matures.

Chez les poissons, les stérö̈des sexuels pourraient donc conditionner la sensibilité olfactive et l'émission de phéromones. Toutefois, les phéromones impliquées dans le comportement sexuel, d'effet immédiat ("releaser pheromones"), apparaissent différentes de ces stéroïdes. Ce fait peut être déduit de l'étude d'AMOURIQ (1965) chez le guppy. Poecilia reticulata, qui notait que la réponse du mâle à l'introduction d'un œestrogène (hexestrol) dans l'eau ne se produisait qu'après plusieurs heures.

\subsubsection{Réserves sur l'action effective des phéromones dans le "homing".}

Chez les poissons, l'existence d'interactions phéromonales complexes ne constitue pas une caractéristique des salmonidés migrateurs et, compte tenu des types d'approche développés (1) chez ces derniers, l'importance de l'action des phéromones dans le "homing" des adultes demeure à préciser. Les aspects remarquables des connaissances acquises en milieu contrôlé devront être confirmés par la mise en place d'expérimentations olfactométriques de terrain, préalables indispensables à la validation de la théorie phéromonale de NORDENG issue de données collectées dans le milieu naturel.

\subsubsection{Imprégnation précoce et relations phéromonales}

Outre l'influence de caractères génétiques sur la précision du retour des salmonidés, le "homing" des reproducteurs (sous contrôle olfactif prédominant) dépendrait donc d'une part de la perception à l'état juvénile des particularités physico-chimiques du milieu de développement (“imprinting" hypothesis) et d'autre part de l'action attractive de substances provenant à la fois des "smolts" en cours de dévalaison et des populations en place de juvéniles ("pheromone" hypothesis).

Même si l'effet attractif des phéromones au moment du "homing" demeure à prouver dans le milieu naturel, les démonstrations présentées à l'appui de chacune des deux hypothèses ne permettent plus de rejeter l'action de l'une au profit de l'autre et la complexité du phénomène laisse à penser que ces deux mécanismes sont impliqués dans l'orientation jusqu'aux sites de fraie originels.

Pendant la phase juvénile, les substances phéromonales produites par les poissons dę mêmes populations devraient, du fait de leur activité à très faible dose sur l'olfaction, contribuer à l'imprégnation chimiosensorielle aux caractéristiques de l'environnement. A l'état reproducteur, la perception chimique de ces mêmes substances à proximité de la rivière constituerait un des vecteurs de l'orientation précise des adultes jusqu'au site originel de développement.

Avec les phéromones, des substances actives sur les comportements interspécifiques à rôle social ou alimentaire (communications allélochimiques), et des composés inorganiques pourraient intervenir dans ce processus de reconnaissance. Nous examinerons le rôle éventuel des substances extra-phéromonales dans le "homing" des salmonidés.

\subsection{Communications allélochimiques}

\subsubsection{Rôle social interspécifique}

Par définition, les phéromones représentent des substances dont l'action sur le comportement est maximale entre individus de même espèce. Ce type de communication n'exclue pas une sensibilité comportementale particulière à la perception chimique de poissons d'autres espèces présentes dans le milieu naturel. Toutefois, les relations chimiques interspécifiques ont fait l'objet de peu d'études approfondies chez les salmonidés et l'origine, la nature et le rôle des substances impliquées demeurent encore à préciser.

HOGLUND et al., (1975) mirent en évidence en fluvarium la réaction d'évitement présentée par les juvéniles d'omble arctique en réponse à l'odeur d'une espèce sympatrique de corégone. Coregonus lavaretus.

(1) Expérimentations électrophysiologiques et olfactométriques en milieu contrôlé. 
Selon les auteurs, ces réactions d'évitement pourraient contribuer chez les poissons à l'élimination d'une espèce de son habitat naturel lors d'une confrontation interspécifique ou à la ségrégation des esnèces à l'intérieur de niches écologiques différentes.

L'éventualité d'interactions chimiques sexuelles entre salmonidés d'espèces différentes a également été étudiée.

Les édudes olfactométriques de PETE (1977) révélèrent une absence d'interattractions entre reproducteurs de saumon chinook et de saumon coho.

Chez Oncorhynchus masou et $O$. rhodorus, HONDA (1982) montra que le comportement sexuel du mâle de chacune de ces espèces n'était produit que par la perception olfactive de la femelle de la même espèce.

Comme pour l'ensemble des phyla étudiés, les phéromones sexuelles de salmonidés se caractériseraient donc par leur spécificité d'action. Le rôle des interactions chimiques interspécifiques demeure inétudié chez le saumon atlantique.

\subsubsection{Rôle alimentaire}

Considérant l'importance de l'alimentation dans la capacité d'apprentissage et de mémorisation des salmonidés (MAC BRIDE et al., 1962 ; ADRON et al., 1973 ; ADRON et MACKIE, 1978), il est par ailleurs probable que des substances d'actions olfactive et gustative émises par les invertébrés constitutifs du régime alimentaire des juvéniles interviennent activement dans le processus d'imprégnation.

Chez des "smolts" de saumon sockeye (O. nerka), MAC BRIDE et al. (1962) ont montré l'importance du régime préalable sur le caractère attractif de différents extraits alimentaires (1) et, après 10 jours de conditionnement à un aliment donné, l'attractivité pouvait être observée en réponse à des doses extrêmement faibles (de l'ordre de $10 \mu \mathrm{g} / \mathrm{l}$ ).

L'action positive de l'alimentation sur l'imprégnation pourrait également se manifester de façon indirecte et constituer l'élément "gratifiant" permettant une sensibilisation particulière des juvéniles vis-à-vis des caractéristiques physico-chimiques globales (substances phéromonales, interspécifiques, minérales et autres) du milieu dans lequel la croissance s'effectue.

Sans exclure l'influence possible d'autres facteurs (2) dans ce phénomène, le faible taux de retour observé après transplantation de "smolts" s'expliquerait dans cette hypothèse par le fait que les poissons n'ont pu établir et "consolider" une telle association au cours de leur très bref séjour dans la rivière.

\subsubsection{Acides aminés et dérivés}

Par leur présence à l'état libre dans le milieu extérieur et par le pouvoir hautement stimulant de ces composés sur les voies olfactives (SUTTERLIN et SUTTERLIN (3), 1971; HARA et al., 1973; BELGHAUG et D $\phi V I N G, 1977$; HARA, 1982) et gustatives (MACKIE, 1982) des salmonidés, les L. acides aminés et dérivés constituent des composés susceptibles d'action dans le mécanisme chimiosensoriel du "'homing".

Compte tenu de la diversité des sources environnementales impliquées dans l'émission des acides aminés et de l'extrême sensibilité comportementale des salmonidés à la nature, à la concentration et aux proportions relatives de ces composés (MACKIE, 1982 ; SHPARKOVSKIY et al., 1981 ; SHPARSKOVSKIY et al., 1983), les acides aminés émis par les organismes présents au niveau dú milieu de développement pourraient selon nous intervenir dans les relations phéromonales et allélochimiques des juvéniles et participer à la formation précoce d'un conditionnement chimiosensoriel, de données spécifiques (4), actives sur l'orientation finale des reproducteurs.

Le schéma théorique ci-contre résume l'influence possible des acides aminés libres du milieu de développement sur les manifestations comportementales de salmonidés migrateurs.

L'attractivité de certains acides aminés sur l'orientation des reproducteurs a été démontrée "in situ" par SHPARKOVSKIY et al. (1981) chez le saumon atlantique et chez le saumon pink. Chez les

(1) Foie ou cœur de bœuf, zooplancton, crevette ou calmar.

(2) Génétique, conditionnement au lâcher.

(3) Seuil d'activité des acides aminés sur l'olfaction du saumon atlantique de l'ordre de $10^{-9} \mathrm{M}^{-}$

(4) Liées à la nature et à l'importance relative des divers peuplements. 


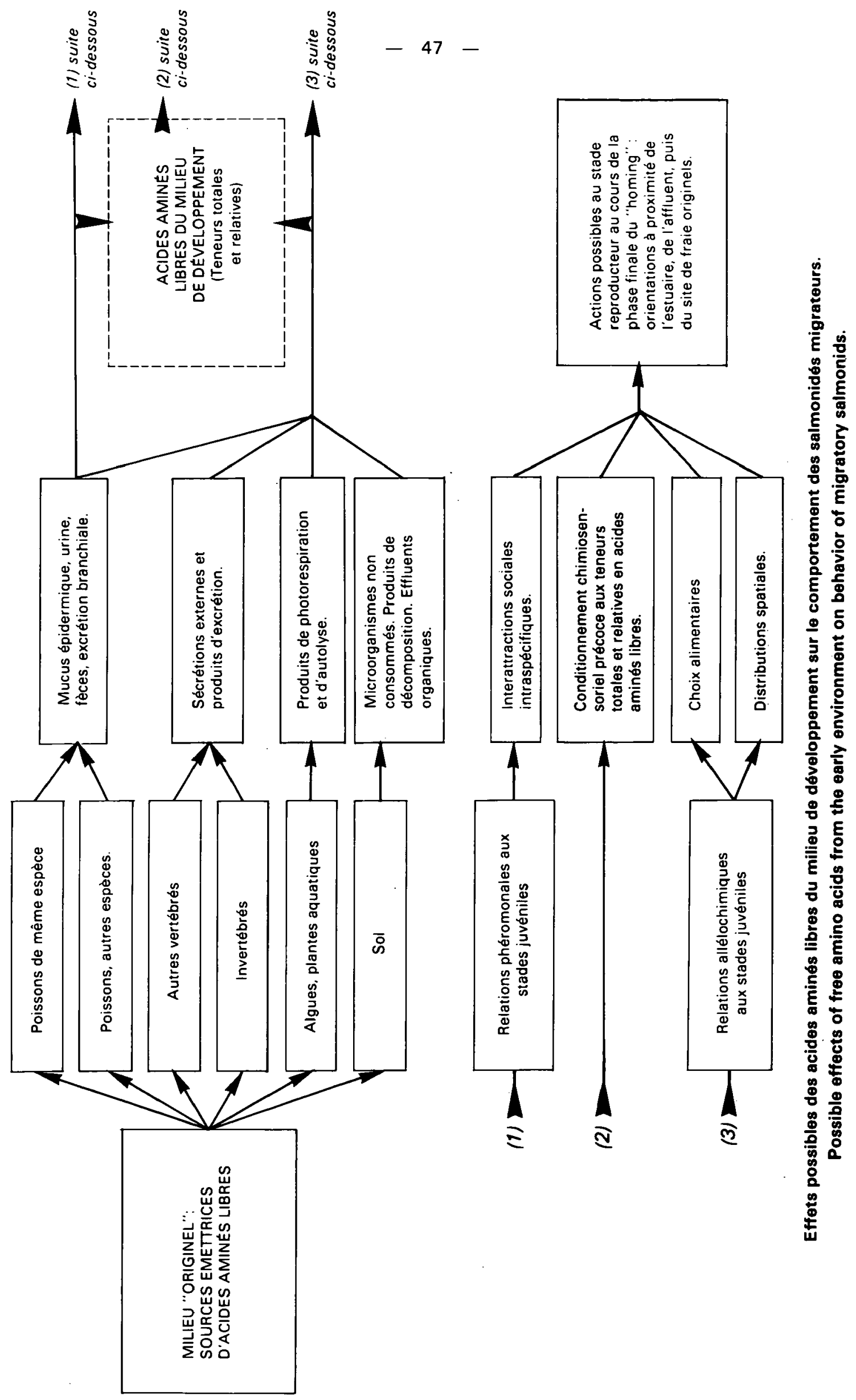


juvéniles, SHPARKOVSKIY et al. (1983) ont également montré les actions individuelles et synergétiques des acides a minés sur les déplacements du saumon atlantique en olfactomètre. Plus récemment, les observations de MEARNS (1985) sur des alevins de cette espèce ont révélé le caractère précoce de la sensibilité comportementale à de faibles doses d'acides aminés.

Le rôle déterminant de la fraction d'acides a minés caractéristique du régime dans la séquence comportementale alimentaire des poissons a été démontré chez de nombreuses espèces (MACKIE, 1982 ; CARR, 1982). L'action éventuelle des acides aminés d'origine intraspécifique ou d'origine allélochimique non alimentaire sur le comportement des salmonidés demeure par contre à préciser.

Les travaux d'IDLER et al. (1956-1961) qui révélèrent que la sérine constituait le principal composé responsable de l'action répulsive de la peau de mammifère sur la remontée migratoire de saumons du genre Oncorhynchus sont à ce titre exceptionnels. L'hypothèse d'une action des acides aminés libres dans les orientations alimentaires et sociales des salmonidés s'appuie plus particulièrement sur les faits suivants, constatés chez différentes espèces de poissons :

- les effets synergétiques des acides aminés dans la séquence comportementale alimentaire,

- l'effet très précis de la nature et de la concentration en acides aminés testés sur l'orientation des déplacements en olfactomètre,

- les variations observées dans les teneurs totales et relatives en acides aminés libres du mucus épidermique ou d'invertébrés d'espèces différentes,

- la présence dans le mucus de hautes teneurs en acides aminés libres permettant leur action stimulante au niveau des voies olfactives (et gustatives) de poissons de même espèce ou d'autres espèces sympatriques.

- l'influence de l'expérience sociale antérieure ou du régime alimentaire préalable sur le contenu en acides aminés libres du mucus épidermique.

Ces faits peuvent être déduits en partie des résultats de CARR et CHANEY (1975), SUTTERLIN (1975), CARR (1976), CARR (1982), ADRON et MACKIE (1978), PAWSON (1977), BRYANT et al., (1978), HARA (1982), DABROWSKI et RUSIECKI (1983), SAGLIO et BLANC (1983), FAUCONNEAU et SAGLIO (1984), HARA et al. (1984), SAGLIO et FAUCONNEAU (1985).

\subsection{Substances inorganiques}

II existe peu d'information sur l'action des substances minérales sur les voies chimiosensorielles ou sur le comportement des salmonidés.

Toutefois, des études olfactométriques et électrophysiologiques menées chez le saumon sockeye (BODZNICK, $1978 a, b, c)$ ont montré que cette espèce était capable de discriminer différentes eaux sur la base de leur teneur en matière inorganique. BODZNICK démontra que des concentrations en ion calcium $\left(10^{-6} \mathrm{M} \mathrm{CaCL}_{2}\right)$ inférieures à celles présentes dans le milieu naturel étaient détectées par l'olfaction et possédaient une action sur l'orientation des alevins en olfactomètre. Ces études soulignent le caractère possible d'une contribution des substances minérales dissoutes dans l'imprégnation olfactive précoce aux caractéristiques du milieu.

Des substances toxiques peuvent aussi présenter à dose faible unè action perturbatrice sur la sensibilité chimique et sur le comportement des salmonidés.

La présence dans le milieu de faibles concentrations de métaux lourds diminue de façon importante le sens gustatif des "parr" chez le saumon atlantique (SUTTERLIN et SUTTERLIN, 1970). De même, une brève exposition au mercure $\left(10^{-4} \mathrm{M} \mathrm{HgCl}_{2}\right.$ pendant 10 secondes) suffit pour inhiber la sensibilité olfactive de cette espèce aux acides aminés pendant plus d'une heure (SUTTERLIN et SUTTERLIN, 1971). Chez la truite arc-en-ciel, SPRAGUE (1968) avait mis en évidence des réactions d'évitement pour une concentration de zinc de $5 \mu \mathrm{g} / \mathrm{l}$.

Les fractions organiques et inorganiques du pétrole sont également susceptibles d'altérer la physiologie et les relations chimiosensorielles des poissons (SYAZUKI, 1964 : BARNETT et TIEWS, 1978 ; WANG et NICOL, 1977 ; HELLSTR $\phi M$ et D $\phi$ VING, 1983).

Les concentrations souvent importantes (sinon croissantes) en substances toxiques au niveau de certaines zones déter minantes de l'orientation des salmonidés migrateurs (estuaires, zones littorales) constituent dès à présent un élément préjudiciable au contrôle olfactif du rhéotropisme (WESTERBERG, 1984 ; WESTERBERG et al., 1985) chez les reproducteurs au cours de la phase finale du "homing".

\section{CONCLUSIONS ET PERSPECTIVES}

Les connaissances acquises sur la sensibilité olfactive et le comportement des Salmonidés migrateurs s'accordent avec l'action probable de substances de différentes origines dans le "homing" de ces poissons. 
Des substances intraspécifiques, de caractéristiques chimiques et d'effets spécifiques au niveau de la population, pourraient contribuer à la précision du phénomène.

Outre ces phéromones, des substances allélochimiques et des composés inorganiques seraient susceptibles de participer au caractère unique des données physico-chimiques du milieu mémorisées au stade juvénile et rendre compte de l'orientation des reproducteurs.

Enfin, rien ne permet d'exclure l'éventualité du rôle du conditionnement intraspécifique précoce sur la sensibilité phéromonale au stade adulte, et donc l'action de substances phéromonales dans le mécanisme de l'imprégnation olfactive précoce.

Le mucus épidermique, le fluide ovarien et le mucus intestinal constituent trois sources importantes de médiateurs d'activité intraspécifique chez les salmonidés.

Tandis que l'action des sécrétions épidermiques sur le comportement social ou du fluide ovarien sur l'attraction du mâle existe chez de nombreux poissons, l'effet attractif du mucus intestinal de juvéniles sur les reproducteürs de même population pourrait constituer un trait plus caractéristique de la communication phéromonale chez les salmonidés.

En terme de composés actifs, les phéromones de ces poissons pourraient être regroupées sous deux classes, des dérivés stéroïdiens d'origine biliaire ou gonadique d'une part et des acides aminés libres du mucus épidermique d'autre part.

Bien que la nature chimique et l'impact éthologique de ces substances demeure encore énigmatique dans le contexte du milieu naturel, les acides (et sels) biliaires et les acides aminés représentent à ce jour les composés les plus actifs sur les voies chimiosensorielles et sur les déplacements (en olfactomètre) de ces poissons.

Le marquage du substrat dû à la présence de composés biliaires dans les fèces des juvéniles pourrait intervenir dans les interactions territoriales précoces et dans le choix des sites de fraie par les reproducteurs. Les seuils d'activité des stéroïdes biliaires relevés au niveau des récepteurs olfactifs et gustatifs des salmonidés s'accordent avec une telle hypothèse.

Néanmoins, l'existence de composés biliaires structurellement différents au sein d'une même espèce ou d'espèces apparentées, les transformations subites par ces substances depuis leur émission dans l'intestin jusqu'à leur perception dans l'environnement aquatique, leur action fine sur le comportement social et sur l'orientation en milieu non confiné constituent autant de questions complexes qui demeurent posées.

Outre le rôle probable des acides aminés libres du mucus épidermique dans les processus de reconnaissance intraspécifique (1), les acides aminés libérés par les autres organismes du milieu pourraient constituer des médiateurs déterminants dans les orientations des poissons aux abords et au niveau même des sites de développement.

Les différences observées dans les teneurs totales et relatives en acides aminés libres d'extraits d'espèces distinctes d'invertébrés ou de poissons (mucus épidermique) permettent de considérer que les acides aminés émis par les proies alimentaires, les conspécifiques et les autres poissons et invertébrés, pourraient non seulement présenter une action sélective sur le comportement (alimentaire, social et territorial) des juvéniles, mais ensemble contribuer à la constitution d'un "bouquet" d'acides aminés, image synthétique (mémorisable) des caractéristiques organiques du milieu de développement.

Les acides aminés émis par les autres vertébrés, les algues, les plantes aquatiques et le sol. d'actions chimiosensorielle et comportementale encore peu explorée, pourraient néanmoins contribuer à la définition unique de ce bouquet.

Afin de contrôler cette hypothèse, des recherches traitant plus particulièrement de l'action conditionnante de ces composés sur l'orientation et sur la réponse alimentaire ou sociale des salmonidés et de l'influence des acides aminés libres du milieu (dont aliment) sur l'odeur corporelle de ces poissons devront être favorisées.

A plus court terme, la période critique de sensibilité olfactive et le rôle éventuel de l'alimentation dans l'établissement d'une mémoire olfactive chez le juvénile de saumon atlantique pourraient être précisés par l'introduction de certaines variantes dans la méthode de conditionnement précoce utilisée par HASLER et al. (1978).

A savoir. pour un même composé (non toxique et d'activité olfactive, acide aminé ou dérivé...). les modes de conditionnement distincts suivants (avant lâcher au stade smolt):

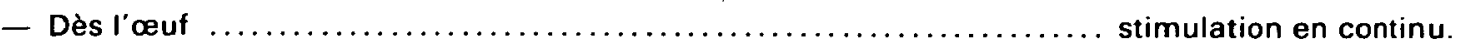

(1) Avec les acides aminés libres du mucus épidermique, les acides aminés excrétés par voie branchiale et digestive pourraient être également impliqués dans ces relations. 
L'approche novatrice d'HASLER nous apparaît comme une base remarquable pour l'étude fine du déterminisme chimiosensoriel du "homing" des salmonidés. En particulier, l'utilisation lors de l'imprégnation de substances naturelles (origines intraspécifiques ou allélochimiques, composés minéraux) devrait conduire à une meilleure évaluation des sources environnementales impliquées dans la migration reproductrice de ces poissons.

\section{REMERCIEMENTS}

Nous remercions I'IFREMER du soutien financier apporté dans le cadre de cette analyse (Contrat INRA/CNEXO/COB 837248 y).

\section{BibLIOGRAPHIE}

ADRON J.W., MACKIE A.M., 1978. Studies on the chemical nature of feeding stimulants for rainbow trout, Salmo gairdneri Richardson. J. Fish. Biol., 12. 303-310.

ADRON J.W., GRANT P.T., COWEY C.B., 1973. A system for the quantitative study of the learning capacity of rainbow trout and its application to the study of food preferences and behaviour. $J$. Fish. Biol., 5, 625-636.

AMOURIO L., 1965. Origine de la substance dynamogène émise par Lebistes reticulatus femelle (Poisson : Poecilidae, Cyprinodontiformes). C.R. Acad. Sci. Paris, 260, 2334-2335.

BAMS R.A., 1976. Survival and propensity for homing as affected by presence or absence of locally adapted paternal genes in two transplanted populations of pink salmon (Oncorhynchus gorbuscha). J. Fish. Res. Bd Can., 33, 2716-2725.

BARNETT J., TIEWS D., 1978. The effects of crude oil and the dispersant, oilperse 43, on respiration and coughing rates in Atlantic salmon (Salmo salar). Can. J. Zool., 56, 307-310.

BELGHAUG R., D $\emptyset$ VING K.B., 1977. Odour thresholds determined by studies of the induced waves in the olfactory bulb of the char (Salmo alpinus L.). Comp. Biochem. Physiol., 57 A, 327-330.

BERTMAR G., TOFT R., 1969. Sensory mechanism of homing in salmonid fish. I. Introductory experiments on the olfactory sense in grilse of Baltic salmon (Salmo salar). Behav., 35, 235-241.

BODZNICK D., 1978 a. Water source preference and lakeward migration of sockeye salmon fry, Oncorhynchus nerka. J. Comp. Physiol., 127, 139-146.

BODZNICK D., $1978 \mathrm{~b}$. Characterization of olfactory bulb units of sockeye salmon with behaviorally relevant stimuli. J. Comp. Physiol., 127, 147-155.

BODZNICK D., $1978 \mathrm{c}$. Calcium ion : an odorant for natural water discriminations and the migratory behavior of sockeye salmon. J. Comp. Physiol., 127, 157-166.

BRAEMER W.. 1960. A critical review of the sun-azimuth hypothesis. Cold Spring Harbor Symp. Quant. Biol., 25, 413-427.

BRANNON E.L., 1972. Mechanisms controlling migration of sockeye salmon fry. Int. Pacif. Salm. Fish. Com. Bull., 21, 1-86.

BRYANT B., ELGIN R., ATEMA J., 1978. Chemical communication in catfish : stress-induced changes in body odor. Biol. Bull., 155, 459.

BUCKLAND F., 1880. Power of smell in salmon. In Natural History of British Fishes, 300-309, Unwin, London.

CALDERWOOD W.L., 1922. The homing of the salmon. Scottish Naturalist, 123-124, 37-43.

CARLIN B., 1968. Salmon Conservation, tagging experiments and migrations of salmon in Sweden. Lecture Series, The Atlantic Salmon Assoc., Montreal.

CARR W.E.S., 1976. Chemoreception and feeding behavior in the pigfish, Orthopristis chrysopterus : characterization and identification of stimulatory substances in a shrimp extract. Comp. Biochem. Physiol., 55 A, 153-157.

CARR W.E.S., 1982. Chemical stimulation of feeding behavior. In Chemoreception in Fishes. HARA T.J. Ed., 259-273. Elsevier Scient. Publ. Comp., Amsterdam. 
CARR W.E.S., CHANEY T.B., 1975. Chemical stimulation of feeding behavior in the pinfish, Lagodon rhomboïdes : characterization and identification of stimulating substances extracted from shrimp. Comp. Biochem. Physiol., 57 A, 437-441.

CHIDESTER F.E., 1924. A critical examination of the evidence for physical and chemical influences on fish migration. J. Exp. Biol., 22, 118-144.

COLLINS G.B., 1952. Factors influencing the orientation of migrating anadromous fishes. $\ddot{U} . \bar{S} . \bar{F} i s h$. Bull., 52, 73, 375-396.

COOPER J.C., 1982. Comment on electroencephalographic responses to morpholine and their relationship to homing. Can. J. Fish. Aquat. Sci., 39, 1544-1546.

COOPER J.C., HASLER A.D., 1976. Electrophysiological studies of morpholine-imprinted cono salmon (O. kisutch) and rainbow trout (S. gairdneri). J. Fish. Res. Bd Can., 33, 688-694.

COOPER J.C.,.SCHOLZ A.T., HORRAL R.M., HASLER A.D., MADISON D.M., 1976. Experimental confirmation of the olfactory hypothesis with homing, artificially imprinted coho salmon $(O$. kisutch). J. Fish. Res. Bd Can., 33, 703-710.

DABROWSKI K., RUSIECKI M., 1983. Content of total and free amino acids in zooplanktonic food of fish larvae. Aquaculture, 30, 31-42.

DENTON J.E., YOUSEF M.K., YOUSEF I.M., KUKSIS A., 1975. Bile acid composition of rainbow trout, Salmo gairdneri. Lipids, 9, 945-951.

DфVING K.B., ENGER P.S., NORDENG H., 1973. Electrophysiological studies on the olfactory sense in char (Salmo alpinus L.). Comp. Biochem. Physiol., 45 A, 21-24.

D $\emptyset$ VING K.B., NORDENG H., OAKLEY B., 1974. Simple unit discrimination of fish odours released by Char (Śalmo alpinus L.) populations. Comp. Biochem. Physiol., 47 A, 1051-1063.

D $\emptyset$ VING K.B., SELSET R., THOMMESEN G., 1980. Olfactory sensitivity to bile acids in salmonidfishes. Acta Physiol. Scand., 108, 123-131.

DUKER G.J., 1981. Instream orientation and species recognition by Pacific salmon. In Salmon and Trout Migratory Behavior Symposium, E.L. BRANNON and E.O. SALO, Editors, 286-295. Univ. Washington, Seattle.

EMANUEL M.E., DODSON J.J., 1979. Modification of the rheotropic behavior of male rainbow trout (Salmo gairdneri) by ovarian fluid. J. Fish. Res. Bd Can., 36, 63-68.

FAUCONNEAU B., SAGLIO Ph., 1984. Protein-bound and free amino acid content in the skin mucus of the European eel, Anguilla anguilla L. Comp. Biochem. Physiol., 77 B, 513-516.

FISKNES B., D $\phi$ VING K.B., 1982. The olfactory sensitivity to group specific substances in Atlantic salmon (Salmo salar L.). J. Chem. Ecol., 8, 1083-1092.

FOSTER N.R., BERLIN W.H., Laboratory evidence of pheromone-mediated spawning site selection in lake trout, Salvelinus namaycush. En preparation. (Abstract presented at the Am. Soc. Ichthyol. and Herp. 60 th Annual Meeting, Fort Worth, Texas).

HARA T.J.; 1967. Electrophysiological studies of the olfactory system of the goldfish, Carassius auratus L. II. Effects of sex hormones on olfactory activity. Comp. Biochem. Physiol., 22 A, 209-225

HARA T.J., 1974. Is morpholine an effective olfactory stimulant in fish ? J. Fish. Res. Bd Can., 31, $1547-1550$.

HARA T.J., 1982. Structure-activity relationships of amino acids as olfactory stimuli. In Chemoreception in Fishes. T.J. HARA ed., 135-157, Elsevier Scient. Publ. Comp., Amsterdam.

HARA T.J., BROWN'S.B., 1979. Olfactory bulbar electrical responses of rainbow trout (Salmo gairdneri) exposed to morpholine during smoltification. J. Fish. Res. Bd Can., 36, 1186-1190.

HARA T.J., BROWN S.B., 1982. Comment on electroencephalographic responses to morpholine and their relationship to homing : reply. Can. J. Fish. Aquat. Sci., 39, 1546-1548.

HARA T.J., MAC DONALD S., 1975. Morpholine as olfactory stimulus in fish. Science (Wash.), 187. 81-82.

HARA T.J., CAROLINA LAW Y.M., HOBDEN B.R., 1975. Comparison of the olfactory response to amino acids in rainbow trout, brook trout and whitefish. Comp. Biochem. Physiol., 45 A, 969-977.

HARA T.J., MAC DONALD S., EVANS R.E., MARUI T., ARAI S., 1984. Morpholine, bile acids and skin mucus as possible chemical cues in salmonid homing : electrophysiological re-evaluation. In Mechanisms of migration in Fishes, MC LEAVE.J.D., ARNOLD G.P., DODSON J.D., NEILL W.H., Eds, 363-378.

HASLER A.D., 1966. Underwater Guideposts. Univ. Wisconsin Press, 155 p. 
HASLER A.D., 1971. Orientation and fish migration. In Fish Physiology, vol. VI, HOAR and RANDALL Eds, 429-510. Academic Press, New York.

HASLER A.D., COOPER J.C., 1976. Chemical cues for homing salmon. Experientia, 32, 1091 -1093.

HASLER A.D., WISBY W.J., 1951. Discrimination of stream odors by fishes and relation to parent stream behavior. Am. Naturalist, 85, 223-238.

HASLER A.D., SCHOLZ A.T., HORRAL R.M., 1978. Olfactory imprinting and homing in salmon. Am. Scientist, 66, 347-355.

HASLEWOOD G.A.D., 1967. Bile salt evolution. J. Lipid Res., 8, 535-550.

HATTINGH J., COETZEE N., 1975. Possible stimuli for social behavior in three species of freshwater fish. Zool. Afric., 10, 219-221.

HELLSTR $\phi M$ T., D $\phi V I N G$ K.B., 1983. Observations of cod behaviour when stimulated with water soluble fractions of oil. In Chemoreception in studies of marine pollution. Reports from a workshop at Oslo, July 13 and 14, 1980. DфVING K.B., Ed., 69-81.

HOAR W.S., 1958. The evolution of migratory behavior among juvenile salmon of the genus Oncorhynchus. J. Fish. Res. Bd Can., 15, 391-428.

HOGLUND L.B.; ASTRAND M., 1973. Preferences among juvenile char (Salvelinus alpinus L.) to intraspecific odours and water currents studied with the fluvarium technique. Rep. Inst. Freshwater Res. Drottningholm, 53, 21-30.

HOGLUND L.B., BOHMAN A., NILSSON N., 1975. Possible odour responses of juvenile Arctic char (Salvelinus alpinus L.) to three other species of subarctic fish. Rep. Inst. Freshwater Res. Drottningholm, 54, 21-35.

HONDA H., 1980. Female sex pheromone of rainbow trout, Salmo gairdneri, involved in courtship behavior. Bull. Jap. Soc. Sci. Fish., 46, 1109-1112.

HONDA H., 1982. On the female sex pheromones and courtship behaviour in the salmonids, Oncorhynchus masou and O. rhodorus. Bull. Jap. Soc. Sci. Fish., 48, 47-49.

IDLER D.R., FAGERLUND U.H.M., MAYOH H., 1956. Olfactory perception in migrating salmon. I. L-serine a salmon repellent in mammalian skin. J. Gen. Phys., 39, 889-892.

IDLER D.R., MC BRIDE J.R., JONAS R.E.E., TOMLINSON N., 1961. Olfactory perception in migrating salmon. II. Studies on a laboratory bio-assay for homestream water and mammalian repellent. Can. J. Biochem. Physiol., 39, 1575-1584.

ISAKSSON A., RASCH T.J., POE P.H., 1978. An evaluation of smolt releases into a salmon and a non-salmon producing stream using two releasing methods. J. Agr. Res. Iceland, 10, 100-113.

LARKIN P.A., 1975. Some major problems for further study on Pacific salmon. Int. North Pacif. Fish. Com. Bull., 32, 3-9.

LILEY N.R., 1982. Chemical communication in fish. Can. J. Fish. Aquat. Sci., 39, 22-35.

MC BRIDE J.R., IDLER D.R., JONES R.E.E., TOMLINSON N., 1962. Olfactory perception in juvenile salmon. I. Observations on response of juvenile sockeye to extracts of food. J. Fish. Res. Bd Can., $19,327-334$.

MACKIE A.M., 1982. Identification of the gustatory feeding stimulants. In Chemoreception in Fishes, HARA T.D. Ed, 275-291. Elsevier Scient. Publ. Comp. Amsterdam.

MADISON D.M., SCHOLZ A.T., COOPER J.C., HORRAL R.M., HASLER A.D., DIZON A.E., 1973. Olfactory hypotheses and salmon migration: a synopsis of recent findings. Fish. Res. Bd Can. Tech. Rep., 414, 35 p.

MEARNS K.J., 1985. Response of Atlantic salmon (Salmo salar L.) yearling to individual L. amino acids. Aquaculture, 48, 253-259.

MIGHELL J., 1975. Some observations of imprinting of juvenile salmon in fresh and saltwater. Symp. Salmon homing (summary notes). Nat. Mar. Fish. Service, Seattle, W.A.

MULLER-SCHWARZE D.; MOZELL M.M., 1977. Chemical signals in Vertebrates. Proceedings of a symposium on chemical signals in vertebrates held at Saratoga Springs, New York. MULLERSCHWARZE D., MOZELL.M.M. ed., 61.0 p., Plenum Press, New York, London:

NEWCOMBE C., HARTMAN G., 1973. Some chemical signals in the spawning behaviour of rainbow trout (Salmo gairdneri). J. Fish. Res. Bd Can., 30, 995-997.

NORDENG H., 1971. Is the local orientation of anadromous fishes determined by pheromones ? Nature, London, 233, 411-413.

NORDENG H., 1977. A pheromone hypothesis for homeward migration in anadromous salmonids. Oikos, 28, 155-159. 
NORTHCOTE T.G., 1962. Migratory behavior of juvenile rainbow trout, Salmo gairdneri; in outlet and inlet streams of Loon Lake, British Columbia: J. Fish. Res. Bd Can., 19, 201-270.

OKADA H., SAKAI D.K., SUGIWAKA K., 1978. Chemical stimulus on the reproductive behavior of the pond smelt. Sci. Rep. Hokkaido Fish Hatchery. 33, 89-99.

OSHIMA K., GORBMAN A., 1968. Modification by sex hormones of the spontaneous and evoked bulbar electrical activity in goldfish. J. Endocr., 40, 409-420.

PARTRIDGE B.L., LILEY N.R., STACEY N.E., 1976. The role of pheromones in the sexual behaviour of the goldfish. Anim. Behav., 24, 291-299.

PAVILLON J.F., VU TAN TUE, 1981. Un aspect de l'absorption des substances organiques dissoutes chez les organismes marins et d'eaux saumâtres: données autoradiographiques aux premiers stades de développement chez Artemia species (ex salina) et Dicentrarchus labrax. Oceanis, 7, 705-718.

PAWSON M.G., 1977. Analysis of a natural chemical attractant for whiting Merlangius merlangus L. and cod, Gadus morhua L., using a behavioral bioassay. Comp. Biochem. Physiol., 56 A, $129-135$.

PECK J.W., 1970. Straying and reproduction of coho salmon (O. kisutch) planted in a Lake Superior tributary. Trans. Am. Fish. Soc., 99, 591-595.

PETE K., 1977. Species-specific odor as a guiding mechanism for local orientation in homing chinook (Oncorhynchus tshawytscha) and coho (O. kisutch) salmon. U.S. Thesis, Univ. Washington, Seatt/e, 55 p.

PIGGINS D.J., BROWNE J., 1982. Sea ranching of salmon in Ireland : present status and some limiting factors. In Sea Ranching of Atlantic Salmon. COST $46 / 4$ workshop. ERIKSSON C., FERRANTI M.P., LARSSON P.O.. Eds, 73-79.

POWERS E.B., CLARK R.T., 1943. Further evidence on chemical factors affecting the migratory movements of fishes, especially the salmon. Ecologr, 24, 109-113.

QUINN T.P., 1985. Salmon homing : is the puzzle complete ? Envir. Biol. Fishes, 12, 4, 315-317.

QUINN T.P., BUSACK C.A.. Chemosensory recognition of siblings in juvenile coho salmon (Oncorhynchus kisutch). Ann. Behav., sous presse.

ROSSELAND L., 1978. Experiences from smolt releases in hydrodynamic regulated rivers. In The biological Effects of Hydrodynamic Regulation in Reservoirs and Salmon Rivers, 243-263. GUNNERD T.B., MELLQUIST P., eds.

ROYCE W.F., SMITH L.S., HARTT A.C., 1968. Models of oceanic migrations of Pacific salmon and comments on guidance mechanisms. Fish. Bull. U.S., 66, 441-462.

RUBEC P.J., 1979. Effect of pheromones on behavior of ictalurid catfish. Ph. D. Thesis, Texas, A.M. University, College Station, T.X., 165 p.

SACQUET E., LESEL R., MEJEAN C., RIOTTOT M., LEPRINCE C., 1979. Absence de transformation bactérienne des acides biliaires chez la truite arc-en-ciel, Salmo gairdneri, élevée à $16^{\circ} \mathrm{C}$. Ann. Biol. Anim. Bioch. Biophys., 19, 385-391.

SAGLIO Ph., 1982. Piégeage d'anguilles (Anguilla anguilla L.) dans le milieu naturel au moyen d'extraits biologiques d'origine intraspécifique. Mise en évidence de l'attractivité phéromonale du mucus épidermique. Acta. Oecol., (Oecol. appl.) 3, 223-231.

SAGLIO Ph., BLANC J.M., 1983. Etude en olfactomètre de la réponse comportementale aux L. acides aminés chez le juvénile de carpe, Cyprinus carpio L. Biol. Behav., 8, 4, 359-370.

SAGLIO Ph., LE MARTRET M.A., 1982. Communication intraspécifique d'origine phéromonale chez le carassin doré immature (Carassius auratus L.). II. Etude en olfactomètre de l'activité comportementale d'extraits d'origine épidermique. Biol. Behav., 3, 221-234.

SAGLIO Ph., FAUCONNEAU B., 1985. Free amino acid content in the skin mucus of goldfish, Carassius auratus L. Influence of feeding. Comp. Biochem. Physiol., sous presse.

SAUNDERS R.L., 1967. Seasonal pattern of return of Atlantic salmon in the northwest Miramichi river, New Brunswich. J. Fish. Res. Bd Can., 24, 21-32.

SAUNDERS R.L., 1977. Sea-ranching. A promising way to enhance populations of Atlantic salmon for angling and commercial fisheries. Int. Atlantic Salmon Foundation. spec. Public. ser., 7, 17-24.

SAUNDERS R.L., 1982. Sea-ranching of Atlantic salmon in Canada. In Sea-Ranching of Atlantic salmon. COST 46/4 workshop. ERIKSSON C., FERRANTI M.P., LARSSON P.O., eds, 5-21.

SAUNDERS R.L., BAILEY J.K., 1980. The role of genetics in Atlantic salmon management. In Atlantic Salmon : its future. WENT A.D.J. ed., 182-200. Proc. 2nd Int. Atl. Salmon Symp. Fishing News Books Ltd., Farnham, Surrey, England. 
SAUNDERS R.L., GEE J.H., 1964. Movements of young Atlantic salmon in a small stream. J. Fish. Res. Bd Can., 21, 27-36.

SCHEER B.T., 1939. Homing instinct in salmon (Onrochynchus). Quater. Rev. Biol., 14, 408-430.

SCHOLZ A.T., COOPER J.C., MADISON D.M., HORRAL R.M., HASLER A.D., DIZON A.E., POFF R.J., 1973. Olfactory imprinting in coho salmon : behavioral and electrophysiological evidence. Proc. Conf. Great Lakes Res., 16, 143-153.

SCHOLZ A.T., HORRAL R.M., COOPER J.C., HASLER A.D., MADISON D.M., POFF R.J., DALYR., 1975. Artificial imprinting of salmon and trout in lake Michigan. Dep. Nat. Res. Fish. Man. Rep., 80, $45 \mathrm{p}$.

SELSET R., D $\oint$ VING K.B., 1980. Behaviour of mature anadromous char (Salmo alpinus L.) towards odorants produced by smolts of their own population. Acta Physiol. Scand., 108, 113-122.

SHPARKOVSKIY I.A., PAVLOVI.D., TROSHICHEVA N.V., KLETS D.V., 1981. Response of pink salmon, Oncorhynchus gorbuscha and Atlantic salmon, Salmo salar, to amino acids during spawning migration. J. Ichthyol., 21, 6, 135-140.

SHPARKOVSKIY I.A., PAVLOV I.D., CHINAKINA A.D., 1983. Behavior of young hatchery-reared Atlantic salmon, Salmo salar (Salmonidae) influenced by amino acids. J. Ichthyol., 23, 4, 140-147.

SIEBERS D., ROSENTHAL H., 197.7. Amino-acid absorption by developing herring eggs. Helgo/änder Wiss. Meeresunters., 29, 464-472.

SOLOMON D.J., 1973. Evidence for pheromone-influenced homing by migrating Atlantic salmon, Salmo salar. Nature London, 244, 231-232.

SPRAGUE J.B., 1968. Avoidance reactions of rainbow trout to zinc sulfate solution. Water Res., 2 , 367-372.

STABELL O.B., 1982. Homing of Atlantic salmon in relation to olfaction and genetics. In Proceedings of the Salmon and Trout Migratory Behavior Symposium, BRANNON E.L., SALO E.D., eds, 238246. Univ. Washington, Seattle.

STABELL O.B., 1982. Detection of natural odorants by Atlantic salmon parr using positive rheotaxis olfactometry. In Proceedings of the Salmon and Trout Migratory Behavior Symposium, BRANNON E.L., SALO E.O., eds, 71-78, Univ. Washington, Seattle.

STABELL O.B., 1984. Homing and olfaction in salmonids : a critical review with special reference to the Atlantic salmon. Biol. Rev., 59, 333-388.

STABELL O.B., SELSET R., 1980. Comparison of mucus collecting methods in fish olfaction. Acta Physiol. Scand., 108, 91-96.

STABELL O.B., SELSET R., SLETTEN K., 1982. A comparative chemical study on population specific odorants from Atlantic salmon. J. Chem. Ecol., 8, 201-217.

STACEY N.E., HOURSTONA.S., 1982. Observations on the spawning and feeding behaviour of captive Pacific herring. Can. J. Fish. Aquat. Sci., 39, 489-498.

STASKO A.B., 1971. Review of field studies on fish orientation. Ann. New York Acad. Sci., 188, $12-29$.

STASKO A.B., SUTTERLIN A.M., ROMMEL S.A., ELSON P.F., 1973. Migration-orientation of Atlantic salmon (Salmo salar L.). In Int. Atlantic salmon Symp. (SMITH M.W., CARTER W.M., eds), 119-137. The Int. Atlantic Salmon Foundation, New York and St Andrews.

SUTTERLIN A.M., 1975. Chemical attraction of some marine fish in their natural habitat. J. Fish. Res. Bd Can., 32, 6, 729-738.

SUTTERLIN A.M., GRAY R., 1973. Chemical basis for homing of Atlantic salmon (Salmo salar) to a hatchery. J. Fish. Res. Bd Can., 30, 985-989.

SUTTERLIN A.M., SUTTERLIN N., 1970. Taste responses in Atlantic salmon (Salmo salar) parr. J. Fish. Res. Bd Can., 27, $1927-1942$.

SUTTERLIN A.M., SUTTERLIN N., 1971. Electrical responses of the olfactory epithelium of Atlantic salmon (Salmo.salar). J. Fish. Res.-Bd Can., 28, 565-572.

SYAZUKI K., 1964. Studies on the toxic effects on industrial wastes on fishes and shell-fishes (in Japanese. English summary). J. Shimonoseki Univ. Fish., 13, 157-211.

TEETER J., 1980. Pheromone communication in sea lampreys (Petromyzon marinus) : implications for population management. Can. J. Fish. Aquat. Sci., 37, 2123-2132.

TODD J.H. ATEMA J., BARDACH J.E., 1967. Chemical communication in social behavior of a fish, the yellow bullhead (/cta/urus natalis). Science, 158, 672-673.

TOFT R., 1975. The significance of the olfactory and visual senses in the behaviour of spawning migration in Baltic salmon (in Swedish). Swedish Salmon Research Inst., Rep. 10. 
WANG R.T., NICOL J.A.C., 1977. Effects of fuel oil on sea catfish : feeding activity and cardiac responses. Bull. Environ. Contam. Toxicol., 18, 170-176.

WARD H.B., 1939. Factors controlling salmon migration. Am. Assoc. Advance Sci. Publ., 8, 60-71.

WESTERBERG H., 1984. The orientation of fish and the vertical stratification at fine and microstructure scales. In Mechanisms of Migration in Fishes. MC LEAVE J.C., ARNOLD G.P., DODSON J.J., NEILL W.H., eds, 179-203. Plenum Publ. Corp.

WESTERBERG H., D $\phi V I N G$ K.B., JOHNSEN P.B., 1985. The role of olfaction in the behavioral responses of Atlantic salmon. Salmo salar L., to hydrographic stratification. Sous presse.

WHITE H.C., 1936. The homing of salmon in Apple River, N.S. J. Biol. Bd Can., 2, 391-400.

WISBY W.J., 1952. Olfactory responses of fishes as related to parent stream behaviour. Ph. D. Thesis, Univ. Wisconsin, Madison, 41 p.

WREDE W.L., 1932. Versuch über den Artduft der Elritzen. Z. Vergl. Physiol., 17, 510-51.9.

YAMAZAKI F., WATANABE K., 1979. The role of sex hormones in sex recognition during spawning behaviour of the goldfish, Carassius auratus L. Proc. Indian Nat. Sci. Acad., B 45, 505-511. 